\title{
A Variational Approach for the Fusion of Exposure Bracketed Pairs
}

\author{
Marcelo Bertalmío and Stacey Levine
}

\begin{abstract}
When taking pictures in a dark scene with artificial lighting, the ambient light is not sufficient for most cameras to obtain both accurate color and detail information. The exposure bracketing feature usually available in many camera models enables the user to obtain a series of pictures taken in rapid succession with different exposure times; the implicit idea is that the user picks the best image from this set. But it is often the case that none of these images is good enough: in general, good brightness and color information are retained from longer exposure settings while sharp details are obtained from shorter ones. In this work we propose a variational method for automatically combining an exposure bracketed pair of images into a single picture that reflects the desired properties of each one. We introduce an energy functional consisting of two terms, one measuring the difference in edge information with the short exposure image and the other measuring the local color difference with a warped version of the long exposure image. The method is able to handle camera and subject motion as well as noise, and results compare favorably with the state of the art.
\end{abstract}

\section{INTRODUCTION}

Exposure bracketing is a setting on many cameras in which a series of pictures is taken in rapid succession using varying shutter speeds. Typically the user picks from this set of images the one that has the best compromise between color information and sharp details. This can be quite useful when taking a photograph under difficult lighting conditions. But often none of these images is good enough: longer exposure times preserve good intensity and color information in the sense that the colors from the actual scene are retained, but may result in blurred images; shorter exposure times preserve sharp details, but these images are usually dark and noisy. Increasing the brightness of the short exposure image (e.g. by global histogram matching with the long exposure image) does not solve the problem: not only is the noise amplified, but the colors do not locally match those of the long exposure

M. Bertalmío is with the Departamento de Tecnologías de la Información y las Comunicaciones, Universitat Pompeu Fabra, Barcelona, Spain, marcelo.bertalmio@upf.edu.

S. Levine is with the Department of Mathematics and Computer Science, Duquesne University, Pittsburgh, Pennsylvania, USA, sel@mathcs.duq.edu. 
image. State of the art denoising techniques can help avoid problems with the noise, but are not equipped to rectify the color issues.

Some of the aforementioned challenges are demonstrated in figure 1. Neither the short nor long exposure image by themselves are acceptable with respect to noise level, sharpness, brightness and color. If we attempt to fuse information from these two images by globally modifying the histogram of the short-exposure image to match the histogram of the long-exposure picture, we obtain a result that, apart from being quite noisy, is off in terms of color with respect to the long-exposure image. In fact, it's not unusual to find per-channel differences of $30 \%$ and higher in some regions. In figure 1 this is particularly noticeable in the color of the brown cloth covering the speaker on the left side of the image, the color of the floor, and the color of the bandmembers' shirts. More examples of this behavior can be seen in section IV, figure 6. Pre-processing the short exposure image using an approach such as non-local means denoising [1] prior to global histogram matching solves the noise problem, but the colors still do not locally match those of the long exposure image.

In this work we propose a variational approach for automatically combining an exposure bracketed pair of images into a single picture that reflects the optimal properties of each one. The method proposed here is formulated so that the colors of the output image match closely those of the original long-exposure image, while retaining details (but avoiding high noise levels) from the short exposure image. An example of the kind of results that can be expected from this model is included in figure 1. The proposed technique is intended for still images, however in the final section we suggest how it can be extended to deal with video which often suffers from the same limitations.

There are a number of related works on fusing sets of photographs. The literature is quite extensive and cannot be covered in its entirety here, but we've tried to highlight some of the work most closely related to the proposed model. To start, Fattal et al. [2] and Yuan et al. [3], [4] propose algorithms for deblurring a long-exposure image with a blurry and non-blurry image pair. Current work on picture deblurring assumes the blur only comes from camera motion and the scene is static. This prevents these methods from being applied to most pictures with human subjects since some amount of local motion is usually unavoidable.

Denoising and deblurring image bursts have also been studied. Multichannel blind denconvolution was addressed by Sroubek and Flusser [5], [6] for grayscale images. Telleen et al. [7] and Buades et al. [8] propose techniques that combine a stack of short-exposure images of the same scene to create a noise-free image. The deblurring work in [5], [6] assumes good image alignment and that the majority of the image scene is static, and the work in [7], [8] assumes each image in the burst is noisy but sharp, so blurring is not an issue. Thus these techniques are not readily applicable to the exposure bracketing problem.

Zhang et al. [9] provide an interesting theoretical analysis demonstrating that multi-image denoising is more reliable than single image deblurring. They also propose a method for generating an HDR image from a series of 


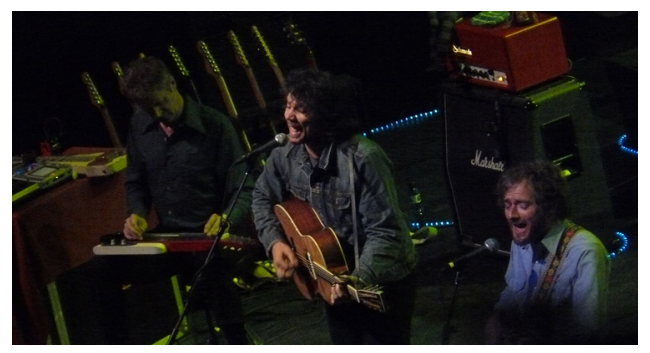

(a) short exposure image

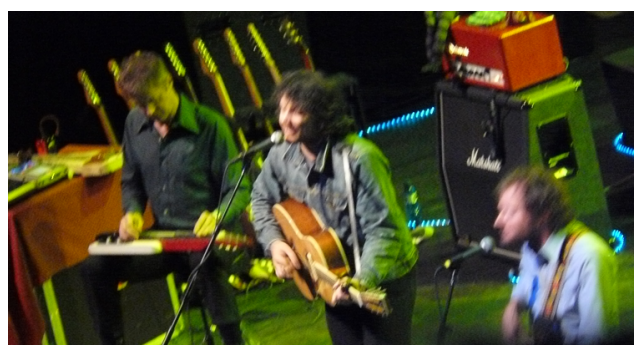

(b) long exposure image

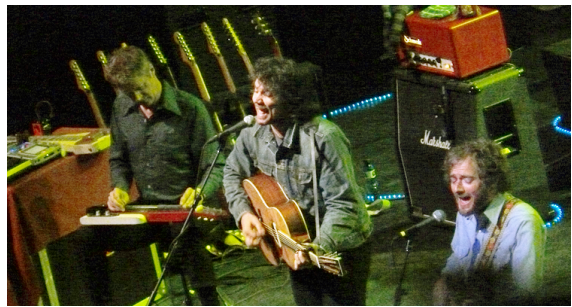

(c) global histogram matching (GHM)

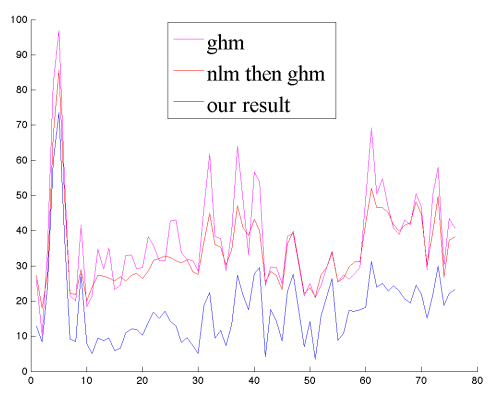

(f) $l_{2}$ error, floor

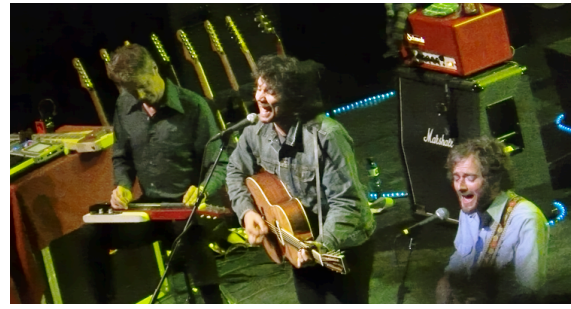

(d) non-local means then GHM

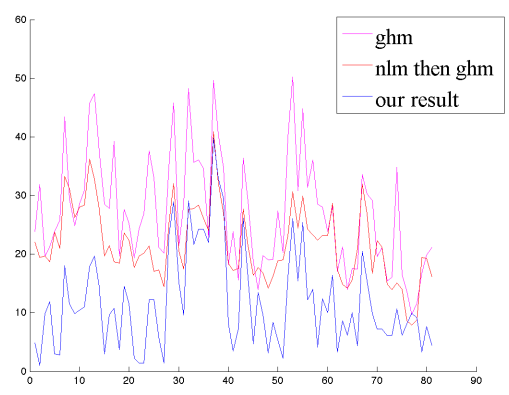

(g) $l_{2}$ error, shirt

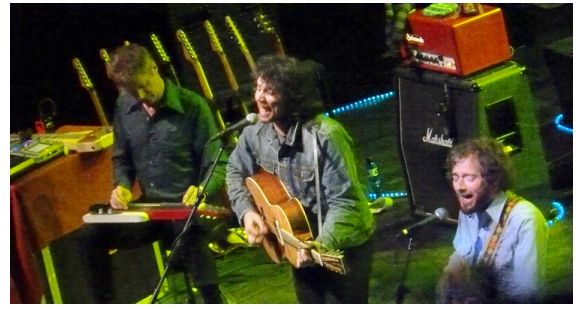

(e) our result

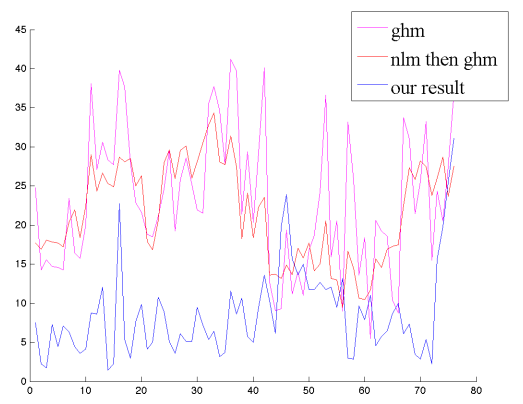

(h) $l_{2}$ error, brown cloth

Fig. 1. Top row: pictures taken with different exposure settings in low light conditions. (a) short exposure image containing sharp objects but noise, low brightness and poor colors. (b) long exposure image containing motion blur but no noise and good colors. Second Row: (c) increasing the brightness of the short exposure image (by global histogram matching with the long exposure image) we can appreciate that it is rather noisy and that its colors do not quite match those of the long exposure image. (d) non-local means (nlm) applied to the short exposure image before global histogram matching. (e) our fusion result. Bottom row: $l_{2}$ errors (with respect to the long exposure image) for typical cross sections comparing the colors of global histogram matching (ghm) (magenta line), global histogram matching after non-local means is performed on the short exposure image (red line), and our result (blue line). (e) section of the floor, (f) section of the left-most band member's shirt $(\mathrm{g})$ section of the brown cloth covering the speaker. The full set of parameters used for this model are listed in Table I under the image name $g c$.

noisy images obtained with a video camera, containing spatially-varying motion caused mostly by camera shake.

This kind of spatially-varying blur is handled quite well using their proposed optical flow based algorithm. The exposure bracketing problem we address here is different in several ways. First, we use a regular handheld camera to obtain two images to be fused instead of a video camera that rapidly collects a large set of images. Furthermore, human subjects are main features of the images we are trying to fuse using the bracketing approach.

The models by Petschnigg et al. [10] and Eisemann and Durand [11] deal with fusing a pair of pictures taken in rapid succession, with and without a flash. Both algorithms use cross bilateral filters (similar to the work by Kopf et al. [12]) to combine the color and detail information from the flash image with the ambient illumination and large scale tonal information from the non-flash image. We demonstrate in section IV, figure 7 how cross bilateral 
filters can be adapted to the fusion of exposure bracketed images by extracting the appropriate information from the long and short exposure images. We will see that for a successful fusion result, this approach requires a rather precise local image alignment of the initial pair; otherwise, visual artifacts become readily apparent. Also, the noise present in the short exposure image is retained or even amplified. The proposed variational approach is able to bypass some of these issues. It should be noted that some recent interesting work on fusing flash/no-flash images has been reported in [13], [14], [15]. Since it's not readily obvious how to adapt these techniques to image pairs that may not involve a flash, we restrict our comparisons with cross-bilateral filtering here.

We could try to solve our image fusion problem with a color transfer technique, where the colors from the long exposure image are transferred to the short exposure one while keeping its details. There is extensive literature on color transfer methods based on histogram modification that goes beyond simple global histogram matching, see for instance Rabin et al. [16], Papadakis et al. [17] and references therein. But these approaches are still global in the sense that they work on the color space and ignore the spatial distributions of color values. This may produce visible artifacts in the processed images. The method by Huang and Chen in [18] is capable of locally transferring color (and brightness) from a blurry, long-exposure image to a sharp, noisy one while allowing for camera and subject motion, but the results sometimes suffer from color leakage, where colors spill outside their corresponding region.

Several techniques have also been proposed for fusing exposure bracketed pairs, the specific problem we address here. Jia et al. [19] propose a technique that combines histogram matching with spatial color matching yielding very good results. However, their algorithm requires the short exposure image be noise-free, a requisite which does not typically hold in practice. Furthermore, the spatial color matching of small regions can be problematic. In [20], Bertalmío and Levine proposed a technique to handle both static and non-static scenes, with camera and/or subject motion. The technique uses the stereo matching algorithm of Cox et al. [21] to extract the color information from the long exposure image and non-local means denoising [1] to suppress noise while retaining sharp details in the luminance of the short exposure image. Although this approach is effective, it requires a number of different steps and parameters that need to be tuned with some care. The technique proposed in the present article is a more concise, mathematically sound approach which improves on the results of [20] (examples can be seen in section IV, figure 7).

Finally, HDR (high-dynamic-range) image fusion is also related to the exposure bracketing problem in that it is intended to fuse images taken with different exposure times, retaining colors and intensity from one or more long exposure images with details from a short exposure image. A number of interesting works have focused on this problem (e.g. [22], [23], [24] just to name a few). Recent approaches such as the one proposed in Tico et.al. [25] are very effective in dealing with this problem, and can handle spatially varying blur which includes human motion. 
In addition, the approach in [25] is quite fast. As we demonstrate in section IV figure 8, the proposed variational method has some advantages of over this kind of HDR approach, including more accurate color transfer from the long exposure image as well as fewer ghosting artifacts. We also discuss there some potential limitations of our model which could inspire future work on the subject.

The paper is laid out as follows. In sections II and III, we discuss the proposed automated algorithm. Numerical results are presented in section IV that demonstrate the effectiveness of the algorithm. In section V, we draw some conclusions and discuss future work.

\section{THE PROPOSED MODEL}

Our problem statement is as follows: given a short exposure image $I_{s}$ and a long exposure image $I_{l}$, we want to create an image $I$ that combines the sharpness of detail of $I_{s}$ and the colors of $I_{l}$. Variational methods provide a concise methodology to tackle several tasks simultaneously. Therefore, we propose minimizing an image functional $E(I)$ with two terms, one measuring the difference in gradient information between $I$ and $I_{s}$, the other measuring the difference in colors between $I$ and $I_{l}$. The minimization of $E(I)$ produces the desired fusion result.

We deal with each color channel independently so in our notation $I, I_{s}, I_{l}$ denote single-valued functions from the image domain $\Omega$ into the real interval $[0,1]$.

\section{A. First energy term: color matching}

The main challenge is to match the colors of our fused image $I$ to those of the long exposure image $I_{l}$. In [26], Sapiro and Caselles proposed a variational formulation for histogram modification. For a given image $I$ they showed that by minimizing the following functional, the histogram of $I$ is equalized:

$$
\begin{gathered}
E_{\text {histogram }}(I):=2 \int_{\Omega}\left(I(x)-\frac{1}{2}\right)^{2} d x \\
-\frac{1}{|\Omega|} \int_{\Omega} \int_{\Omega}|I(x)-I(y)| d x d y
\end{gathered}
$$

where $|\Omega|$ is the size of the image domain and $x=\left(x_{1}, x_{2}\right), y=\left(y_{1}, y_{2}\right) \in \Omega$. They also suggest extending this type of histogram modification to more general gray-value distributions, and to consider locality. We have adapted this idea to our setting. To ensure $I$ has the same histogram as $I_{l}$, the functional we should minimize is:

$$
\begin{gathered}
E_{h, I_{l}}(I):=\frac{2}{|\Omega|} \int_{\Omega} \int_{\Omega}\left|I(x)-I_{l}(y)\right| d x d y \\
-\frac{1}{|\Omega|} \int_{\Omega} \int_{\Omega}|I(x)-I(y)| d x d y
\end{gathered}
$$


This can be understood as follows. The size of the lower level sets of an image $I$ can be measured by the grayscale transformation

$$
H_{I}(\mu):=\frac{1}{|\Omega|} \int_{\Omega} \operatorname{sign}^{+}(\mu-I(y)) d y
$$

where $\operatorname{sign}^{+}(a)=1$ if $a>0, \operatorname{sign}^{+}(a)=\frac{1}{2}$ if $a=0$, and $\operatorname{sign}^{+}(a)=0$ if $a<0$. Notice that $H_{I}(\mu)$ is the value of the normalized cumulative histogram of $I$ at the level $\mu$. Therefore, given an image $I_{l}$, if $H_{I}(\mu)=H_{I_{l}}(\mu)$ for all $\mu \in[0,1]$ (and each of the level sets of $I$ and $I_{l}$ has zero area), then $I$ and $I_{l}$ will have the same histograms. In Appendix A we show that the first variation of the energy in (2) is

$$
\begin{gathered}
\delta E_{h, I_{l}}(I)=\frac{4}{|\Omega|} \int_{\Omega} \operatorname{sign}^{+}\left(I(x)-I_{l}(y)\right) d y \\
-\frac{4}{|\Omega|} \int_{\Omega} \operatorname{sign}^{+}(I(x)-I(y)) d y .
\end{gathered}
$$

Therefore, an image $I$ satisfying $\delta E_{h, I_{l}}(I)=0$, that is, a minimizer of (2), will have the same histogram as $I_{l}$.

Furthermore, since $\int_{\Omega} \operatorname{sign}^{+}(\mu-I(y)) d y$ gives the total number of pixels in $I$ with values below $\mu$, performing gradient descent on the energy in equation (2) yields the following update formula:

$$
I_{t}(x, t)=4\left(p(x, t)-p_{l}(x, t)\right)
$$

where at each pixel $x, p(x, t)$ is the percentage of pixels in the image $I$ with values below $I(x, t)$ and $p_{l}(x, t)$ is the percentage of pixels in the image $I_{l}$ with values below $I(x, t)$. So upon convergence, $I_{t}=0$ and the histograms of $I$ and $I_{l}$ coincide.

But this is a global histogram modification procedure, i.e. the color values are modified regardless of their spatial distributions, and as such it suffers from the shortcomings common in global histogram matching methods as mentioned in the introduction. Figure 2(d) shows another example of this: the yellow and blue colors in the shirt have values that are shifted 5 to 12 percent from the corresponding colors in the long exposure image. Introducing locality into the histogram matching term should alleviate some of these issues:

$$
\begin{gathered}
E_{h, \omega, I_{l}}(I):=\frac{2}{|\Omega|} \int_{\Omega} \int_{\Omega} \omega(x, y)\left|I(x)-I_{l}(y)\right| d x d y \\
-\frac{1}{|\Omega|} \int_{\Omega} \int_{\Omega} \omega(x, y)|I(x)-I(y)| d x d y
\end{gathered}
$$

where $\omega(x, y)$ is a decreasing function of the distance $\|x-y\|$ (in our case $\omega$ is a Gaussian of standard deviation $\sigma$ pixels). The combination of locality and histogram modification in a variational formulation for color and contrast enhancement applications was introduced in Bertalmío et al. [27]. But there is still a problem using the energy in equation (5). If $\sigma$ is large, just as before we have global histogram matching. If $\sigma$ is small, ghosting artifacts appear wherever there is motion since the histograms of the corresponding regions may be too different. Figure 
2(e) shows an example of this: artifacts are clearly visible around the arm, on the fingers and on the shirt-sleeve. To solve this we introduce a kind of "motion compensation" into the functional:

$$
\begin{gathered}
E_{h, \omega, \tilde{I}_{l}}(I):=\frac{2}{|\Omega|} \int_{\Omega} \int_{\Omega} \omega(x, y)\left|I(x)-\tilde{I}_{l}(y)\right| d x d y \\
-\frac{1}{|\Omega|} \int_{\Omega} \int_{\Omega} \omega(x, y)|I(x)-I(y)| d x d y
\end{gathered}
$$

where $\tilde{I}_{l}$ is a warped version of $I_{l}$ that keeps the colors of $I_{l}$ but whose shapes have been deformed so that its geometry is close to that of $I_{s}$. How you construct this warped $I_{l}$ is not essential to the algorithm. In our case, we have tested using the stereo matching algorithm of Cox et al. [21] as in [20] and also a commercial warping software package [28], but for example one could use the automatic warping/morphing technique of Shinagawa and Kunii [29], or perform warping based on an estimation of the optical flow (see Papenberg et al. [30] and references therein). Figure 2(f) shows the result obtained minimizing this last equation, and the previously seen artifacts have disappeared.

Let us point out that Delon and Desolneux [31] propose a related model for the problem of flicker elimination in movies, where they use optical flow computation and average the midway histogram of corresponding patches along the sequence. Also, very recently Papadakis et al. [17] proposed a closely related variational model for the problem of histogram transfer of two or more color images: their energy functional is composed of three terms, one that tends to approach the histograms of the original images, and two other terms that tend to maintain the original colors and geometry. Through the energy minimization process the images evolve and their histograms approach a common, midway histogram. But this process is spatially global, not local, i.e. pixels with the same color values are modified in the same way, regardless of their location in the image.

\section{B. Second energy term: gradient direction information}

The fusion result $I$ should retain the sharp details of $I_{s}$. This can be rephrased in the following way: the level lines of $I$ should match those of $I_{s}$ so that the edges in both images coincide. Approximating the direction of the edges by the orthogonal gradient direction, the intended matching of level lines may be achieved by locally matching the gradient direction:

$$
E_{g, I_{s}}(I):=\int_{\Omega}\left(|\nabla I(x)|-\nabla I(x) \cdot \frac{\nabla I_{s}(x)}{\left|\nabla I_{s}(x)\right|}\right) d x .
$$

Ballester et al. [32] first proposed this type of energy in the context of image inpainting: given a normalized field $\theta$ (where the normalized gradient of $I_{s}, \frac{\nabla I_{s}(x)}{\left|\nabla I_{s}(x)\right|}$, plays the role of $\theta$ ), minimizing an energy such as (7) propagates the values of $I$ along this field. Notice that we use the normalized gradient of $I_{s}$ so the strength of the gradient does not come into play. $I$ should have the same level lines as $I_{s}$ (given by the gradient direction), but not the 


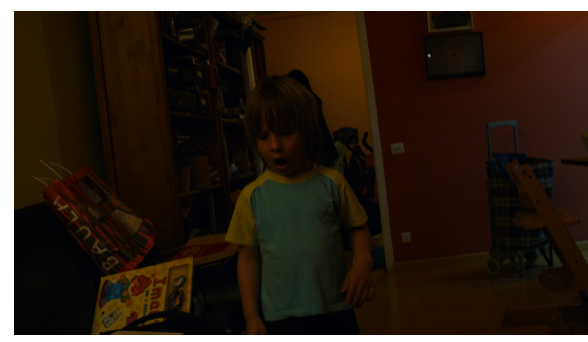

(a)

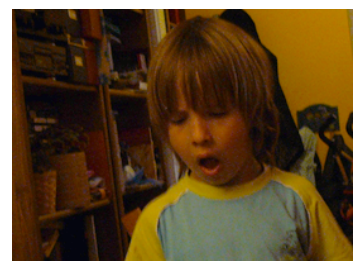

(d)

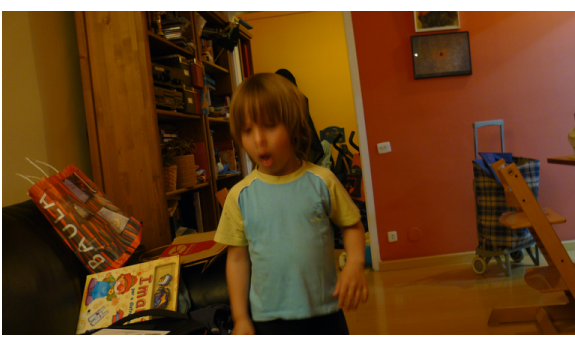

(b)

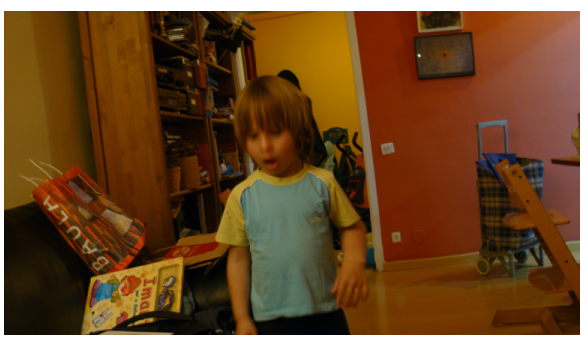

(c)

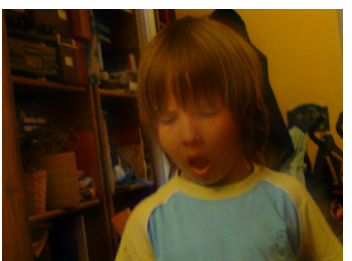

(e)

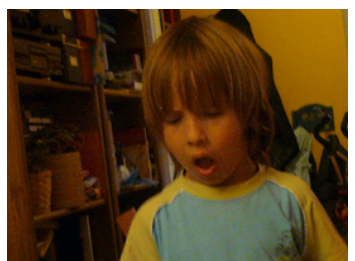

(f)

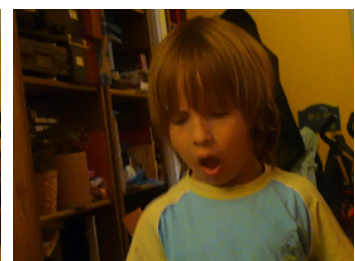

(g)

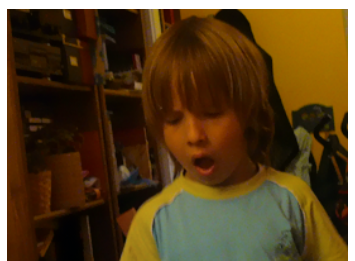

(h)

Fig. 2. (a) short exposure image. (b) long exposure image. (c) long exposure image warped to be locally registered with the short exposure image (warping performed with [28]). (d) result obtained with the global histogram matching of eq. (2). Notice that colors in shirt don't exactly match corresponding colors in the long exposure image. (e) result obtained with the local histogram matching of eq. (5). Ghosting artifacts (due to inter-image motion) are visible around eyes and nose of the child, and next to his left shoulder. (f) result obtained using local histogram matching and a warped long exposure image, eq. (6). Colors are correct and there isn't any ghosting, but there is some noise. (g) result obtained with full model, eq. (8). (h) result obtained with full model, eq. (8), but warping as in [20]. In all cases involving local histogram matching, the kernel width has been $\sigma=17$. The full set of parameters used for this model are listed in Table I under the image name lucas.

same brightness and contrast (given by the gradient magnitude) since $I_{s}$ is poor in these respects.

But one key observation to be made is the following: global histogram modification does not alter the level lines, and while local histogram modification does modify the level line structure of the image it does so in a way that is usually unnoticeable (depending on the width of the kernel and the amplitude of the discontinuities present in the image: see [27] for a rigorous discussion of this).

So, if the sole purpose is to ensure that the level lines of $I_{s}$ are preserved, it suffices with the local histogram term and this second energy term is not necessary. But underexposed images normally suffer from significant amounts of noise, and it is in this regard where the term of (7) becomes useful. The minimization of (7) involves two curvature terms, which are further discussed in section III, and we have found that the value $\epsilon \simeq 0$ used in the numerical computation of these curvatures to avoid division by zero has a regularizing effect on the final image result: if $\epsilon$ is increased, the amount of smoothing also increases but the edges are still kept sharp and smooth regions are not flattened. We believe this is a novel result. This denoising effect is demonstrated in section IV, figure 4, and discussed further in section III.

Remarkably, for the noise levels appearing in real-life situations like the ones depicted in the images in this article, the denoising power of our formulation is comparable to that of state of the art algorithms such as non-local means [1], but at a fraction (one tenth, in our experiments) of the computational cost. Apart from this practical application, the regularizing effect of $\epsilon$ suggests that we may denoise an image by denoising its curvature: this is 
also a novel approach, with interesting theoretical as well as applied implications, that we are currently exploring and will be reported elsewhere [33].

\section{Total energy}

We propose minimizing the following energy, which combines a gradient direction term and a local histogram matching term:

$$
E(I)=E_{g, I_{s}}(I)+\lambda E_{h, \omega, \tilde{I}_{l}}(I),
$$

where $E_{g, I_{s}}$ and $E_{h, \omega, \tilde{I}_{l}}$ are defined above in equations (7) and (6) respectively, $\lambda>0$ is a weighting parameter, and the energy $E$ is minimized over all possible fused images $I$. Figure 2(g) shows the result obtained with this full model: there are no artifacts and the noise has been reduced due to the gradient direction term of equation (7) (the denoising effect of this term is further discussed in the following section). Figure 2(h) also shows the result obtained with this full model, but with a different warping method for the local histogram matching (yielding nearly identical results).

Remark 1: Since $E_{g, I_{s}}(I) \geq 0$ and $E_{h, \omega, \tilde{I}_{l}}(I) \geq-\frac{2 \lambda}{|\Omega|} \int_{\Omega} \int_{\Omega} \omega(x, y)\left|\tilde{I}_{l}(y)\right| d x d y$, the energy defined in equation (8) is bounded below and thus has a minimizing sequence $\left\{I^{j}\right\}$ such that $E\left(I^{j}\right) \rightarrow \inf E(I)$. Since the sequence is bounded in $B V(\Omega)$ (the space of functions of bounded variation), it has a subsequence that converges to a global minimizer of (8). Uniqueness is not so readily determined.

\section{IMPLEMENTATION}

Computing the Euler-Lagrange equation associated with the energy defined in equation (8) and using a gradientdescent approach we obtain the following update equation for $I$ :

$$
\begin{gathered}
I_{t}(x, t)=\kappa_{I}(x, t)-\kappa_{I_{s}}(x) \\
+\frac{2 \lambda}{|\Omega|} \int_{\Omega} \omega(x, y) \operatorname{sign}(I(x, t)-I(y, t)) d y \\
-\frac{2 \lambda}{|\Omega|} \int_{\Omega} \omega(x, y) \operatorname{sign}\left(I(x, t)-\tilde{I}_{l}(y)\right) d y
\end{gathered}
$$

where $\kappa_{I}(x, t)=\nabla \cdot\left(\frac{\nabla I(x, t)}{\sqrt{|\nabla I(x, t)|^{2}+\epsilon}}\right)$ is the Euclidean curvature of the level line of $I$ at location $x$ and evolution time $t, \kappa_{I_{s}}(x)=\nabla \cdot\left(\frac{\nabla I_{s}(x)}{\sqrt{\left|\nabla I_{s}(x)\right|^{2}+\epsilon}}\right)$ is the curvature of the level line of the given short exposure time image $I_{s}$ at location $x$, and sign is the sign function (see Appendix B for details). Note that the curvature terms are regularized with a parameter $\epsilon>0$. The initial condition is $I(x, 0)=\hat{I}_{s}(x) \forall x \in \Omega$, where $\hat{I}_{s}(x)$ is the result of globally matching the histogram of $I_{s}$ to the histogram of $I_{l}$. Only the computation of $\hat{I}_{s}$ is performed in the $R G B$ color 
space: all remaining computations are done in $\mathrm{YCbCr}$, which is a de-correlated color space and therefore more suited for color transfer operations, as pointed out by Reinhard et al. [34], [35].

The denoising effect of the curvature term can be interpreted as follows. If the gradient magnitude is small, $|\nabla I|^{2}<\epsilon$, then $\kappa_{I}=\nabla \cdot\left(\frac{\nabla I}{\sqrt{|\nabla I|^{2}+\epsilon}}\right)$ behaves like the Laplacian, i.e. isotropic diffusion is performed and noise is reduced due to this local averaging. This is the precise situation with the noise in non-edge regions of $I_{s}$, since it is a rather dark image so the noise has very small gradient magnitude. If the regularizing parameter $\epsilon$ is increased, the amount of smoothing also increases but the edges are still kept sharp and smooth regions are not flattened, unlike with TV denoising (this behavior will be illustrated in section IV, figure 4).

For the computation of the curvature terms we use the classical approach of Rudin et al. [36], i.e. an explicit, forward-time scheme with alternating forward-backward spatial differences. The integral terms involve a convolution with the Gaussian kernel $\omega$, and therefore computing these terms explicitly would have a computational complexity of order $O\left(N^{2}\right)$ where $N$ is the number of pixels. To avoid this problem, following the approach introduced in Bertalmío et. al. [27] we approximate the sign function with a polynomial and compute the convolutions using the Fast Fourier Transform (FFT), thus reducing the complexity to $O(N \log N)$.

In a direct implementation of (9), the explicit scheme for the curvature term would impose a very small time step for our iterations, while for the local histogram term a much larger time step suffices. For this reason, we have split the evolution equation and alternate between curvature and histogram terms, running $T_{\kappa}$ steps of

$$
I^{n+1}(x)=I^{n}(x)+\Delta t_{\kappa}\left(\kappa_{I}^{n}(x)-\kappa_{I_{s}}(x)\right)
$$

after each step of

$$
\begin{aligned}
I^{n+1}(x)= & I^{n}(x)+\Delta t\left(\frac{2 \lambda}{|\Omega|} \int_{\Omega} \omega(x, y) \operatorname{sign}\left(I^{n}(x)-I^{n}(y)\right) d y\right. \\
& \left.-\frac{2 \lambda}{|\Omega|} \int_{\Omega} \omega(x, y) \operatorname{sign}\left(I^{n}(x)-\tilde{I}_{l}(y)\right) d y\right)
\end{aligned}
$$

where $\Delta t$ is much larger than $\Delta t_{\kappa}$.

\section{EXPERIMENTS AND COMPARISONS}

We ran our algorithm on several pairs of exposure bracketed images taken with a consumer camera. For each experiment we have alternated one iteration of (11) $(\Delta t=0.1)$ and one iteration of (10) $\left(\Delta t_{\kappa}=0.0025\right)$, i.e. $T_{\kappa}=1$, for a total of 25 steps each. Table I lists the particular values for the parameters $\epsilon$ and $\sigma$ for all the examples. Although they have been chosen so as to optimize the visual quality of the results, the choice has not involved extensive trial and error, instead it is rather intuitive: higher scene motion requires a larger $\sigma$, and higher noise requires a larger $\epsilon$. 
TABLE I

PARAMETER VALUES FOR EACH IMAGE.

\begin{tabular}{|l|c|c|}
\hline Image & $\sigma$ & $\epsilon$ \\
\hline Fig. 1: gc & 17 & $1 \times 10^{-4}$ \\
\hline Fig. 2: lucas & 50 & $2.5 \times 10^{-5}$ \\
\hline Fig. 3, row 1: banda & 100 & $5 \times 10^{-4}$ \\
\hline Fig. 3, row 2: bfn & 100 & $3.5 \times 10^{-5}$ \\
\hline Fig. 3, row 3: sadako & 17 & $7 \times 10^{-5}$ \\
\hline Fig. 3, row 4: dell & 10 & $5 \times 10^{-5}$ \\
\hline Fig. 5: tweedyB & 100 & $1 \times 10^{-4}$ \\
\hline
\end{tabular}

Using non-optimized code on a dual processor $1.87 \mathrm{GHz}, 3 \mathrm{~Gb} \mathrm{PC}$, the computational cost for each $770 \times 430$ image is about 21 seconds (this is not taking into account the warping of $I_{l}$ to obtain $\tilde{I}_{l}$ as it is not part of our algorithm, but a warping time of a few seconds for this image size is reasonable). This is a reduction of $80 \%$ in computation time with respect to a straightforward, non-split implementation of the evolution equation (9), which takes around two minutes per image. Most of the computational cost (around 19.5 seconds) is due to the histogram matching, but since the computation of this term is based on the FFT, and hardware implementations of the FFT are popular and much faster than their software counterparts, we can expect that a hardware implementation of our technique would drastically reduce the computational cost. The gradient term takes around 1.5 seconds per image, which is one order of magnitude less than the non-local means denoising [37] time of around 16 seconds, for a comparable output.

\section{A. Examples}

Typical results can be found in figures 1,2, and 3 for a variety of real-life scenarios. In general we notice that the colors are correctly transferred from $I_{l}$, noise is reduced, and there is no color leakage. Note that the original images, taken without a tripod, have some challenging characteristics that our algorithm is able to handle. In particular, it compensates for a wide range of human motion. Although in some of the images the subjects move very little (e.g. the third row in figure 3), this small amount of motion causes noticeable blurring due to the exposure time required to properly capture the colors. Again, since these are very common situations, methods that require static scenes and still subjects wouldn't be practical. We should also point out that the use of flash was not allowed to obtain the image in the second row of figure 3 (another rather common scenario), which prevents the direct use of fusion methods involving flash/no-flash image pairs. However, we do address how to adapt cross bilateral filters (the basis of some flash/no-flash models) to our problem in section IV-D.

\section{B. Gradient direction term}

Figure 4 shows the effect of varying $\epsilon$, the regularizing parameter introduced to avoid division by zero in the computation of the curvature. As $\epsilon$ increases, the smoothing effect is more pronounced, but edges are still kept 

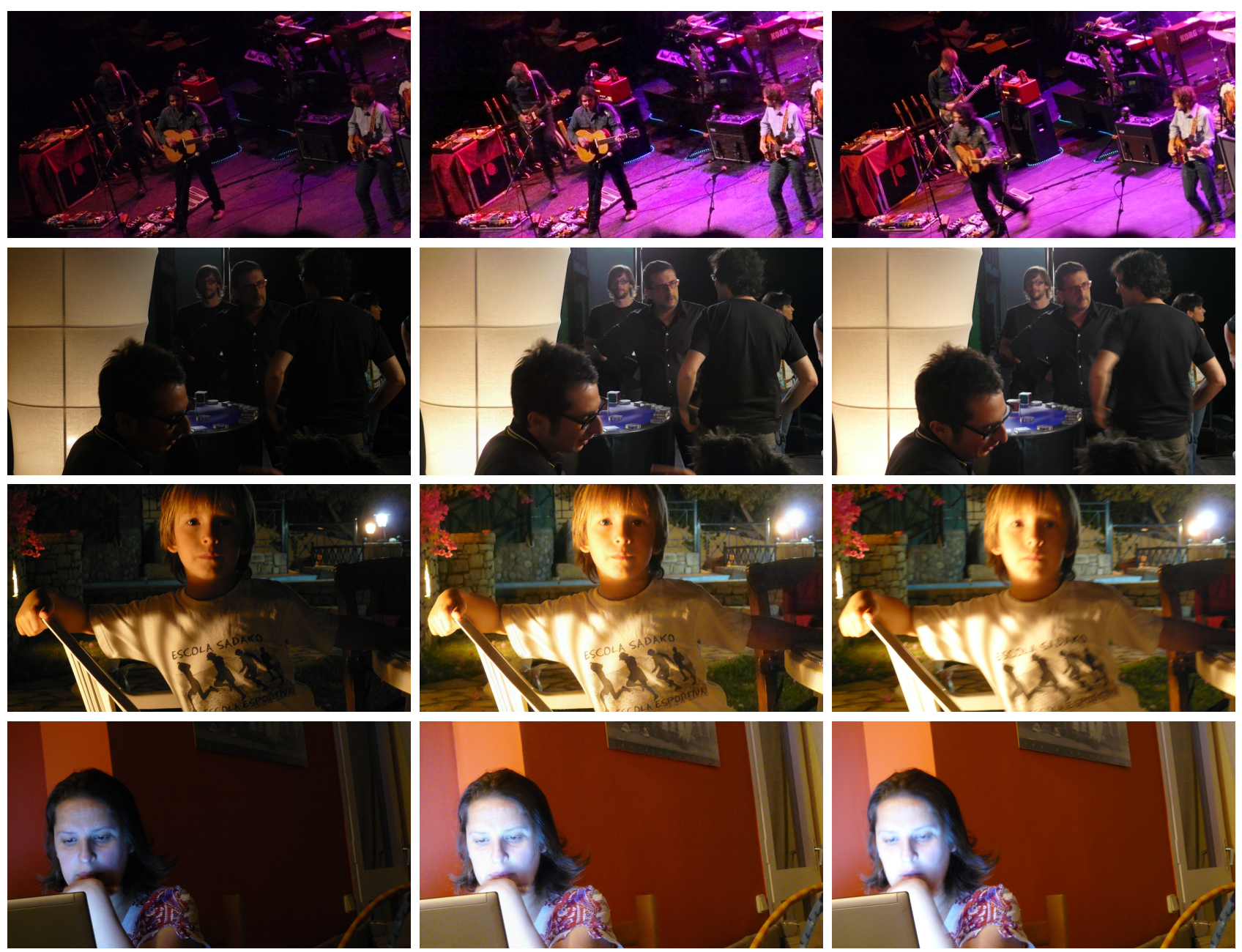

Fig. 3. Left column: short exposure images. Right column: long exposure images. Middle column: our fusion result. From top to bottom: images banda, bfn, sadako, dell. The full set of parameters used can be found in Table I.

sharp and smooth regions are not flattened, unlike one would get with TV denoising.

\section{Histogram matching term}

In general we have observed that for typical examples, the performance of the proposed model is generally invariant to the choice of warping method (see for example, figures $2(\mathrm{~g})$ and $(\mathrm{h})$ ). However, there are still cases in which too much motion and/or occlusion may cause problems with the warping which in turn results in color artifacts after processing (see figure 5). This is still a challenge when using the proposed model. Since most of the guitar is occluded in one view, the fusion result presents wrong colors on the guitar and the singer's jacket when we use a small value for parameter $\sigma$. The simplest solution would be to just increase the value of $\sigma$, as we can see in the same figure. A larger $\sigma$ implies less locality in the histogram matching and therefore less susceptibility to errors in the image warping. The trade-off is of course that as the histogram matching becomes less local the colors are modified in a more global way and they may drift noticeably from the desired result, as figure 2 demonstrated. 


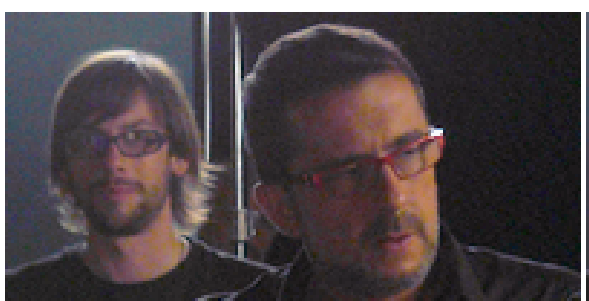

(a)

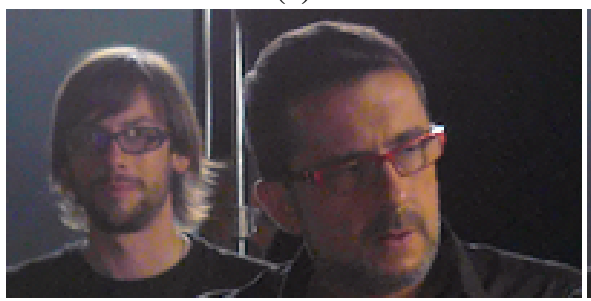

(d)

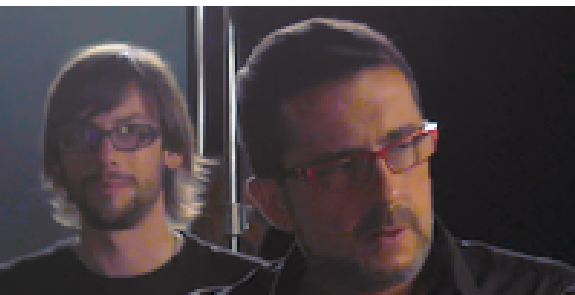

(b)

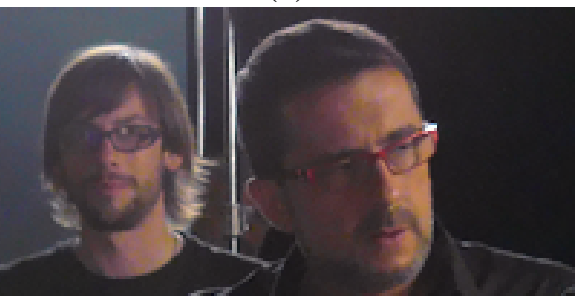

(e)

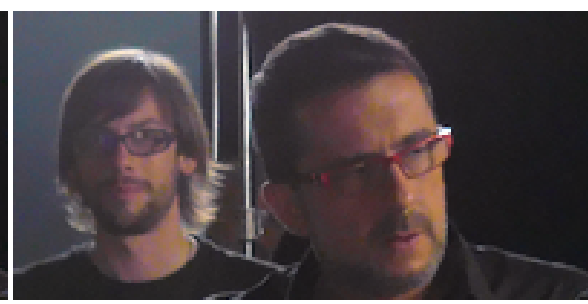

(c)

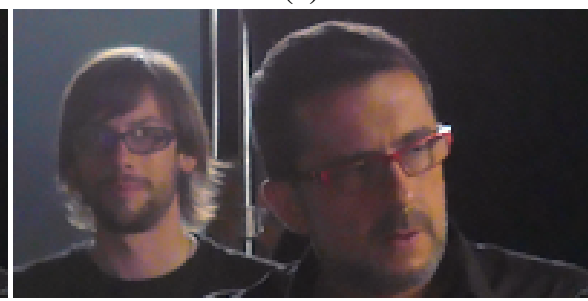

(f)

Fig. 4. Close of the $b f n$ image in the second row of figure 3. Top: (a) Our result without the gradient term. (b) Non-local means denoising of the result in (a), using a value of 4 for the estimated standard deviation of the noise. (c) Our result using both terms, $\epsilon=1.5 \cdot 10^{-4}$. Bottom: effect of varying $\epsilon$. From left to right: $\epsilon=1 \cdot 10^{-5}$ (d), $1 \cdot 10^{-4}$ (e), $1 \cdot 10^{-3}$ (f).
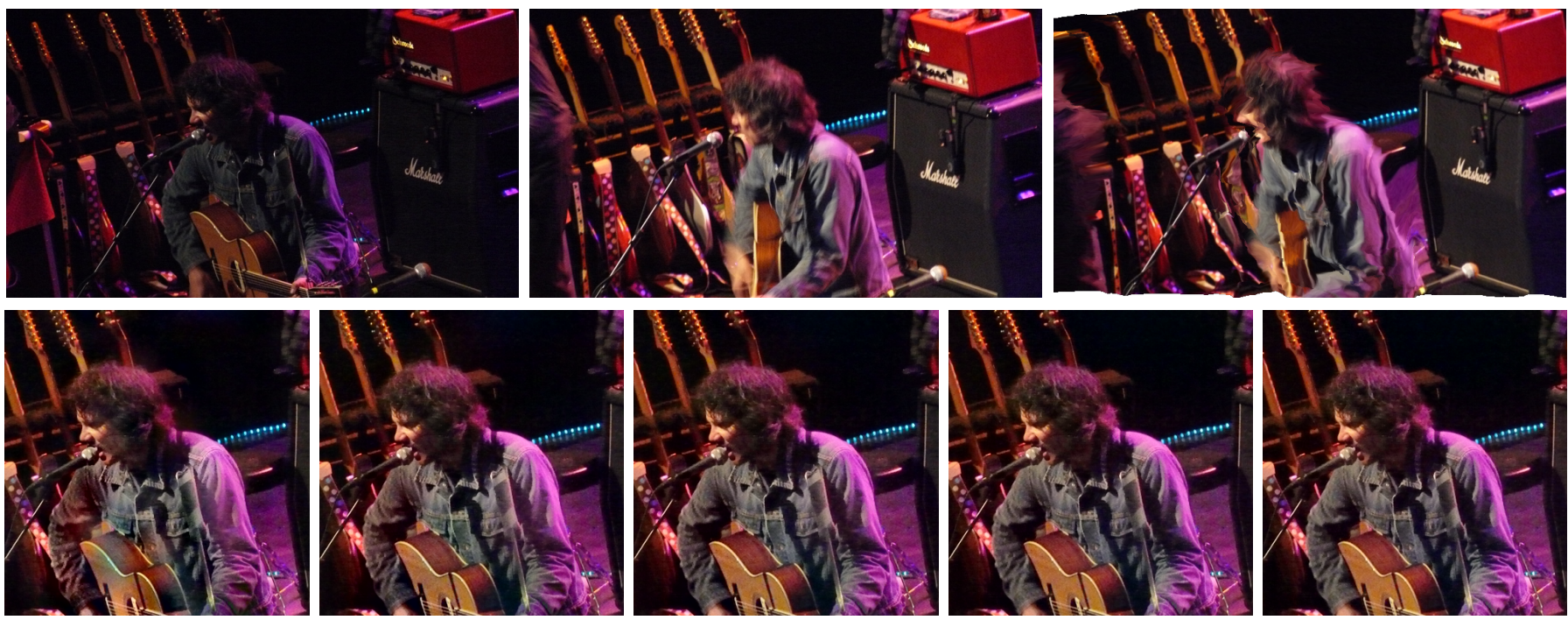

Fig. 5. Effect of varying $\sigma$. Top row: short exposure image (left), long exposure image (middle), warping of long exposure image (right). Bottom row: fusion results for increasing values of $\sigma$ : from left to right, $\sigma=25,50,75,100,125$. The full set of parameters used for this model are listed in Table I under the image name tweedyB.

\section{Comparisons with other methods}

1) Color transfer techniques: For comparison, we start by pointing out that if we try a simple global histogram matching procedure for our problem, the results suffer from visible shifts in the colors as we can see in figures 1 and 2, with more detailed examples included in the third column of figure 6. Papadakis et.al. [17] proposed a variational technique in which two images are modified to have the same histogram; if one of the images is fixed, the method performs color transfer. However, this approach is still global in nature and thus has some difficulties handling dark images and the extreme differences between histograms we allow for in the bracketed image pairs (a work-around for noisy and low-key images is also suggested in [17]). Examples of what one might expect from 
this approach are contained in the fourth column of figure 6.

As a basis for comparison, figure 6 also contains the results of the proposed variational approach, along with the cumulative $l_{2}$ errors of the RGB components of the middle row of each image (with respect to the warped long exposure image) in the fifth and sixth columns respectively.
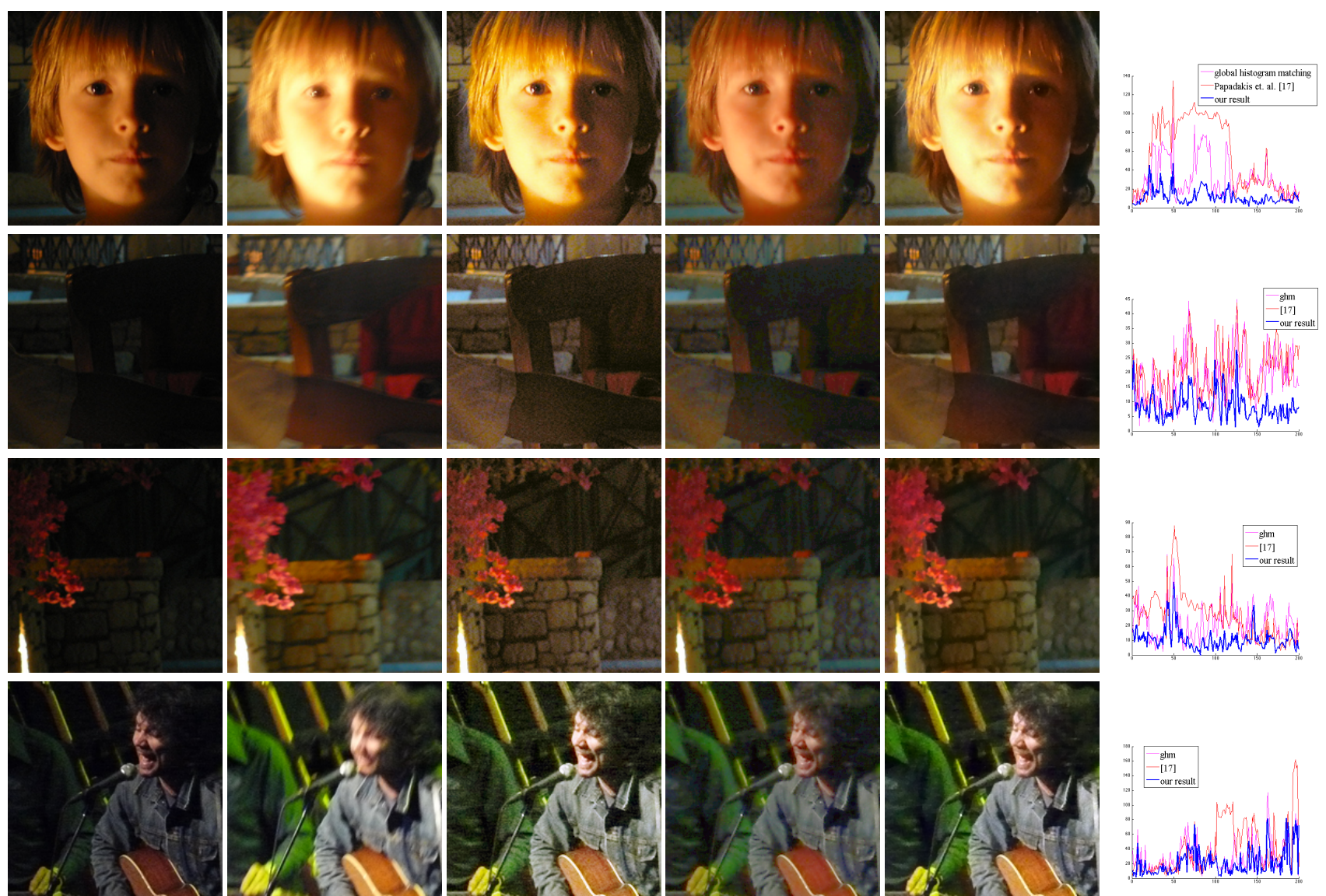

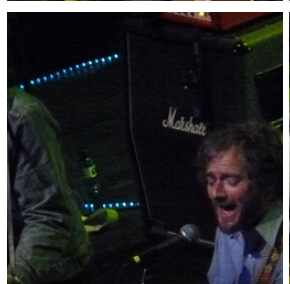

(a)

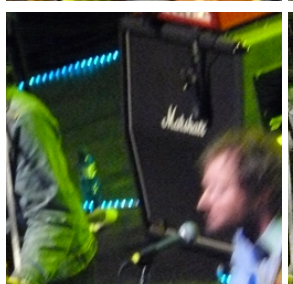

(b)

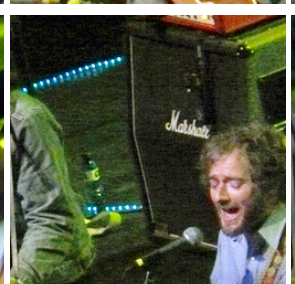

(c)

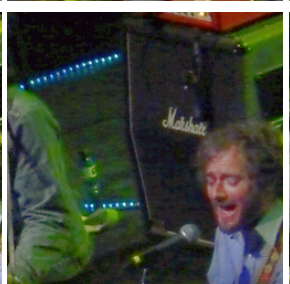

(d)

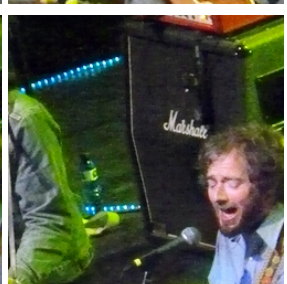

(e)

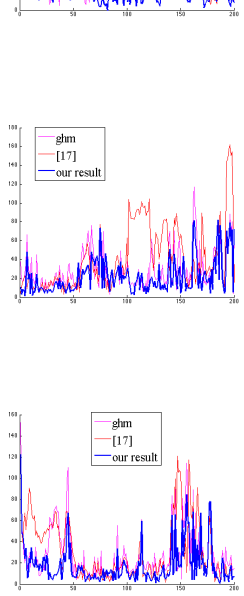

(f)

Fig. 6. Images illustrating the challenge in using global color transfer techniques: $(a)$ : short exposure image, $(b)$ : long exposure image, $(c)$ : global histogram matching, $(d)$ : Papadakis et.al. [17], $(e)$ : proposed variational approach, $(f)$ : $l_{2}$ errors for the middle row of each image result with respect to the warped long exposure image (magenta $=$ global histogram matching, red $=[17]$, blue $=$ our result). The first three images are cropped from the sadako image in the third row of figure 3 and the last two are from the $g c$ image in figure 1.

2) Cross Bilateral Filters: In the third column of figure 7 we compare our results with those of using cross bilateral filters as in the flash/no-flash fusion work in Petschnigg et al. [10] and Eisemann and Durand [11]. The information obtained from each image in the flash/no-flash pair is different than the information obtained from the exposure bracketed $I_{s} / I_{l}$ pair. Therefore, in order to make the fairest possible comparison (i.e. getting the best possible result from the cross bilateral filter approach) we have adapted the bilateral filtering fusion to our problem 

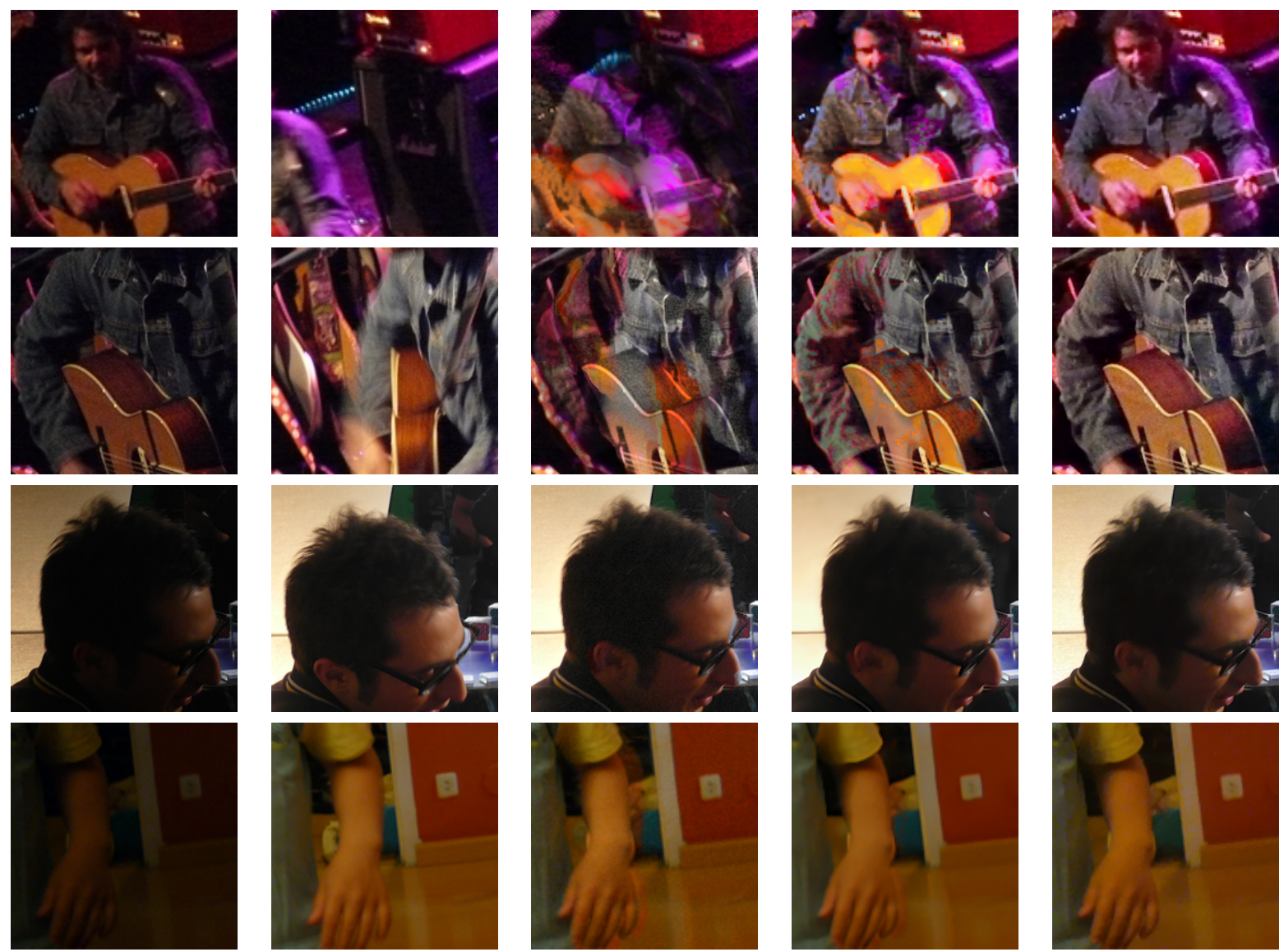

(c)

(d)

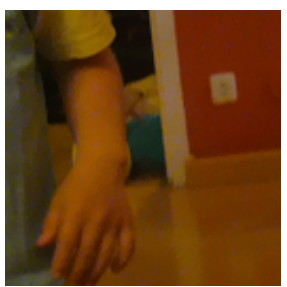

(b)

(e)

Fig. 7. Images with varying amounts of motion between frames: $(a)$ : short exposure image, $(b)$ : long exposure image, $(c)$ : cross-bilateral filters, $(d)$ : result from [20], $(e)$ : proposed variational approach. The first two images are cropped from the banda image, the third is from the $b f n$ image (both from figure 3), and the last is from the lucas image in figure 2.

in the following way.

First, we take the luminance channel of $I_{s}$ and apply bilateral filtering to it. The difference between the filtered image and the original will be the detail layer of the final fusion result. Second, we take the warped long exposure image $\tilde{I}_{l}$. Its colors will be the colors of the final fusion result. We take its luminance channel and apply bilateral filtering to it. The filtered image will be the large scale layer of the final fusion result. Finally, these layers are combined in a straightforward manner [11]: adding the large scale and detail layers we obtain the luminance of the fusion result, while the colors come directly from the source image $\tilde{I}_{l}$. We can anticipate a shortcoming of this type of technique: if the warping isn't precise, the colors will be transferred from incorrect locations. For this reason, cross bilateral filters are fairly sensitive to the particular choice of image alignment method employed, while the results obtained through our method do not vary greatly when switching from one warping method to another, as it was observed in figure 2. Aside from the higher noise level, ghosting and color leakage is more prevalent when using bilateral filters. This is readily seen in close-up images in figure 7; the subject's head has virtually disappeared from the first image, color leakage is prevalent in the second and third images, and ghosting around the subject's 
arm can be found in the last image. Also, we should point out that by increasing $\sigma$, our method can handle artifacts due to incorrect warping (at the expense of shifting colors) as was demonstrated in figure 5. However, the cross bilateral filter has no innate mechanism for achieving this, so results using bilateral filters are only as accurate as the warping.

3) Exposure Fusion: We recall from the introduction that most work in the literature on image fusion of pairs of photographs have limitations that make them impractical for our particular problem: they may require little or no camera and/or subject motion, no noise in the short exposure image, or no blur caused by human motion. There are very few works with no restrictions on camera/object motion and presence of blur, e.g. Jia et al. [19], Huang and Chen in [18], Bertalmío and Levine in [20].

The method in [19] requires the short exposure image to be noise-free, a requisite which does not usually hold in practice, and the spatial color matching of small regions is problematic. In [18] the results often suffer from color leakage. So we compare our results with those obtained with the method presented in [20], which can be found in the fourth column of figure 7 . We can see that there is significant color leakage in the first two images, some amount of color leakage on the subject's face and substantial leakage on the arm in the background of the third image, and ghosting around the subject's arm in the last image.

4) HDR Techniques: Finally, in figure 8 we compare our model with the method of Tico et al. [25], which creates HDR (High Dynamic Range) images by fusing a set of two or more exposure-bracketed images, allowing for both camera and subject motion. In their technique, images are photometrically aligned and fusion is performed in the wavelet domain for the luminance channel. In the chroma channels, colors are directly transferred from static pixels only. The model in [25] works well and one of its particular strengths is its fast computation time. Several critical differences illustrate important properties of our model which is why we included this comparison here.

It is important to point out that in [25] the color information is obtained as a weighted average of the color from all input images, although color information from the short exposure image should only play a significant role in pixels for which the long exposure image is determined to have too much blur. This would explain why the result in figure 8(h) appears a bit darker than the long exposure image 8(f). We plotted the cumulative $l_{2}$ error of the RGB components of the result from Tico et.al. [25] and our result against the warped long exposure time images for fairly flat regions (the ceiling, the chair, and the subject's shirt) to get a better comparison of the color information in each image.

Figure 8 also shows that while the method from Tico et al. [25] has a good denoising performance, the result can have noticeable ghosting artifacts due to the motion of the subject, as seen on the rim of the glasses, which appear duplicated. Our result is noisier but no new edges are introduced due to the geometry-preserving properties of both terms of our energy functional. However, this result also shows some of the limitations of our approach that 
could be improved upon. In our model we assume that the geometric information is better preserved in the short exposure picture, but in this particular example the static regions have better details in the long exposure image, whereas they are too noisy in the short exposure shot.

We should note that the HDR problem is different in nature in that the goal is to fuse three or often more photographs to obtain some sort of intermediate color information. So it's not surprising that this kind of approach wouldn't directly translate to solving the exposure bracketing problem addressed in this work.

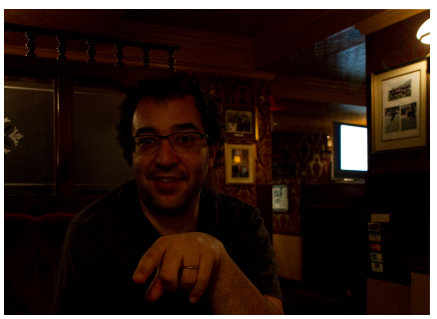

(a) short exposure image

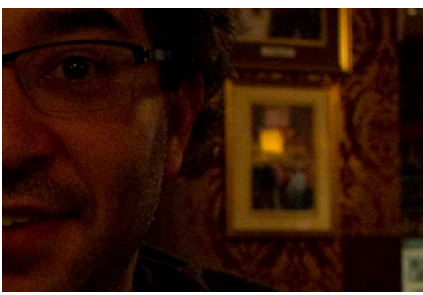

(e) short exposure image

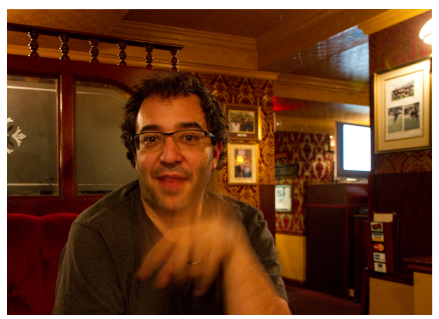

(b) long exposure image

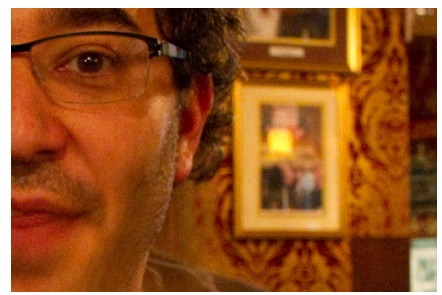

(f) long exposure image

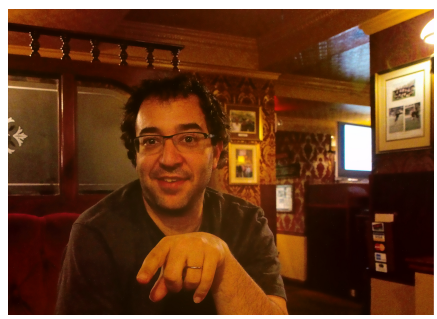

(c) our result

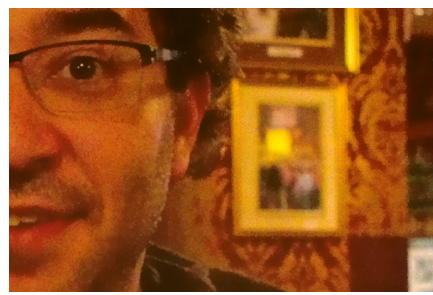

(g) our result

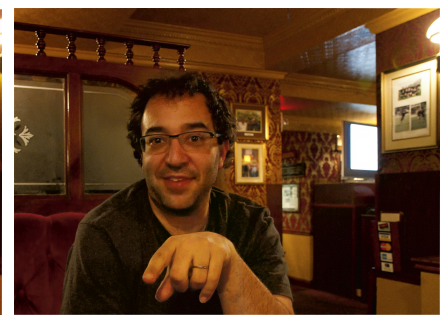

(d) Tico et al.

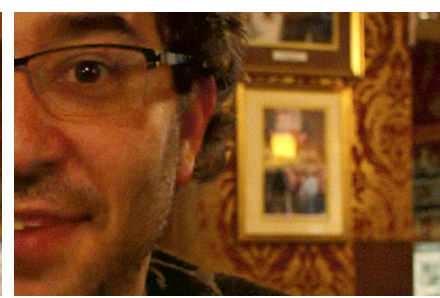

(h) Tico et al.

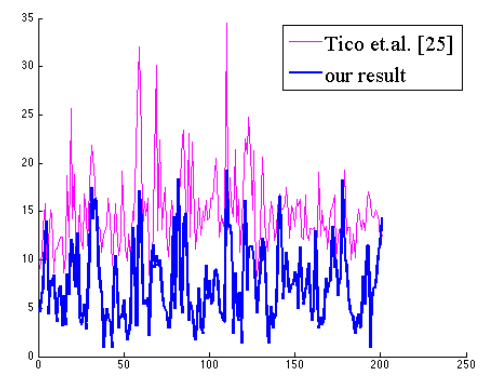

(i) $l_{2}$ error: ceiling

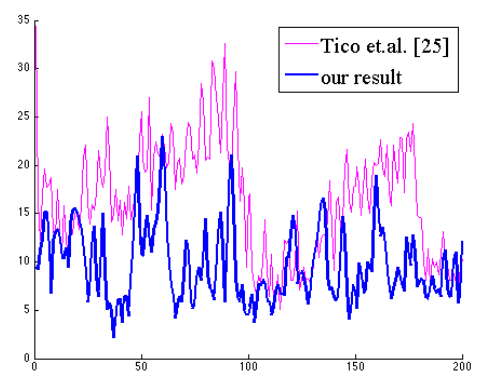

(j) $l_{2}$ error: chair

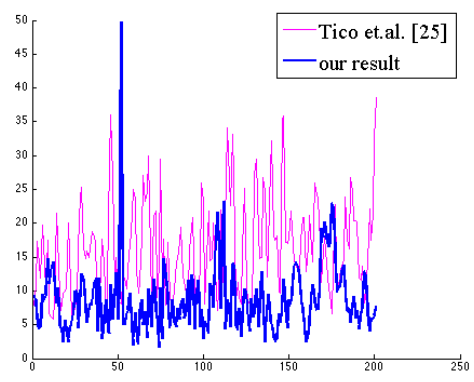

(k) $l_{2}$ error: shirt

Fig. 8. Comparison with Tico et al. [25]. (a),(b): pictures taken with different exposure settings in low light conditions (original images courtesy of M. Tico and K. Pulli); (c): our fusion result $\left(\sigma=400, \epsilon=1 \cdot 10^{-4}, T_{\kappa}=8\right.$ ); (d): result from Tico et al. [25]; (e)-(h): details demonstrating how our method better preserves colors from the long exposure image, but also has the limitation that given a dark image taken by a static camera, the geometry in the short exposure image may retain too much noise and the details in the long exposure image could be of use; (i)-(k) cross-sectional $l_{2}$ errors of the RGB components with respect to the warped long exposure time image.

\section{CONCLUSIONS AND FUTURE WORK}

In this work we proposed a variational formulation for automatically combining an exposure bracketed pair of images in such a way that yields an image retaining the desired qualities from each one. The method can handle camera and subject motion and reduces the noise present in the short exposure image. Furthermore, the colors in 
the fused image locally match those of the long exposure image without suffering from the usual color leakage artifacts common in the literature. The final results compare favorably with the state of the art.

The main shortcomings of the proposed technique are its computational cost, and the dependence of the results on the warping method used in the case of severe occlusions. Also, our method preserves the geometric information from the short exposure image, but it may be the case that better gradient information is available in the longexposure image: for instance, in a very dark scenario and using a static camera, the geometry will be corrupted by noise in the short exposure image while it will be better preserved in the long exposure image, as it was shown in fig. 8. In this case it would be interesting to try to combine geometric information from both sources. Conversely, the long-exposure image may be overexposed in some regions which are well exposed and contain good color information in the short-exposure image, therefore we would need here to combine the color information from both images. We are currently investigating how to overcome these limitations.

This work has an interesting potential extension to video processing. In particular, if the first frame $F_{0}$ of a video is obtained with a long exposure time and the remaining frames, $F_{i}$ for $i=1, \ldots, T$, with short exposure, we can apply the proposed method in the following way. First apply the method described in section II to the pair $\left(F_{0}, F_{1}\right)$ obtaining $\tilde{F}_{1}$, then successively to each pair $\left(\tilde{F}_{n-1}, F_{n}\right)$ obtaining $\tilde{F}_{n}$. In theory, the color from $F_{0}$ should be transferred to all remaining frames, while they still retain a good level of detail. But occlusion can be a problem as soon as the frames are too far apart. Furthermore, as the geometric configuration of the scene changes over time so will the global illumination configuration. This will be the subject of further work.

\section{ACKNOWLEDGMENTS}

We would like to thank Prof. Vicent Caselles for his suggestions and discussions, Pierre Jasmin of RE:Vision Effects, Inc. for kindly providing us with the Twixtor warping results, Marius Tico and Kari Pulli for providing us with their test images and results for comparison, Marius Tico and Edoardo Provenzi for kindly running comparison tests, and the Institute for Mathematics and Applications (IMA) in which the authors were visitors in 2011. We also thank the reviewers for their careful reading of the original submission and productive feedback which greatly improved the manuscript. The first author acknowledges partial support by AACC project, reference TIN201115954-E. The second author is funded in part by NSF-DMS \#0915219.

\section{APPENDIX A}

\section{THE FIRST VARIATION OF (2)}

In order to make this article more self-contained we include here the derivation of the first variation of (2), the energy corresponding to the histogram matching term, though we would like to point out that the following proofs are based on very similar proofs which can be found in [26], [27]. 
Setting

$$
\operatorname{sign}^{+}(a)=\left\{\begin{array}{ll}
1 & a>0 \\
\frac{1}{2} & a=0 \\
0 & a<0
\end{array} \quad \text { and } \quad \operatorname{sign}(a)= \begin{cases}1 & a>0 \\
0 & a=0 \\
-1 & a<0\end{cases}\right.
$$

we show that the first variation of (2)

$$
\begin{gathered}
E_{h, I_{l}}(I)=\frac{2}{|\Omega|} \int_{\Omega} \int_{\Omega}\left|I(x)-I_{l}(y)\right| d x d y \\
-\frac{1}{|\Omega|} \int_{\Omega} \int_{\Omega}|I(x)-I(y)| d x d y,
\end{gathered}
$$

is

$$
\begin{gathered}
\delta E_{h, I_{l}}(I)=\frac{2}{|\Omega|} \int_{\Omega} \operatorname{sign}\left(I(x)-I_{l}(y)\right) d y \\
-\frac{2}{|\Omega|} \int_{\Omega} \operatorname{sign}(I(x)-I(y)) d y .
\end{gathered}
$$

Note that the equality $\operatorname{sign}(a)=2 \operatorname{sign}^{+}(a)-1$ results in (3).

Because of the absolute value functions in $E_{h, I_{l}}(I)$, to show (12) we first consider an approximate differentiable functional such as

$$
\begin{gathered}
E_{h, I_{l}}^{\epsilon}(I)=\frac{2}{|\Omega|} \int_{\Omega} \sqrt{\left|I(x)-I_{l}(y)\right|^{2}+\epsilon^{2}} d x d y \\
-\frac{1}{|\Omega|} \int_{\Omega} \sqrt{|I(x)-I(y)|^{2}+\epsilon^{2}} d x d y
\end{gathered}
$$

The first variation of $E_{h, I_{l}}^{\epsilon}(I)$ with respect to the increment $\delta I(x)$ is

$$
\begin{gathered}
\delta E_{h, I_{l}}^{\epsilon}(I, \delta I)=\lim _{t \rightarrow 0^{+}} \frac{1}{t}\left[E_{h, I_{l}}^{\epsilon}(I+t \delta I)-E_{h, I_{l}}^{\epsilon}(I)\right] \\
=\frac{2}{|\Omega|} \int_{\Omega} \int_{\Omega} \frac{I(x)-I_{l}(y)}{\sqrt{\left|I(x)-I_{l}(y)\right|^{2}+\epsilon^{2}}} \delta I(x) d x d y \\
-\frac{1}{|\Omega|} \int_{\Omega} \int_{\Omega} \frac{I(x)-I(y)}{\sqrt{|I(x)-I(y)|^{2}+\epsilon^{2}}}(\delta I(x)-\delta I(y)) d x d y
\end{gathered}
$$

Interchanging the roles of $x$ and $y$ we have that $\int_{\Omega} \int_{\Omega} \frac{I(x)-I(y)}{\sqrt{|I(x)-I(y)|^{2}+\epsilon^{2}}} \delta I(y) d x d y=-\int_{\Omega} \int_{\Omega} \frac{I(x)-I(y)}{\sqrt{|I(x)-I(y)|^{2}+\epsilon^{2}}} \delta I(x) d x d y$, so the first variation of $E_{h, I_{l}}^{\epsilon}$ at $I$ is

$$
\begin{gathered}
\delta E_{h, I_{l}}^{\epsilon}(I)=\frac{2}{|\Omega|} \int_{\Omega} \frac{I(x)-I_{l}(y)}{\sqrt{\left|I(x)-I_{l}(y)\right|^{2}+\epsilon^{2}}} d y \\
-\frac{2}{|\Omega|} \int_{\Omega} \frac{I(x)-I(y)}{\sqrt{|I(x)-I(y)|^{2}+\epsilon^{2}}} d y .
\end{gathered}
$$

Letting $\epsilon \rightarrow 0$ results in the first variation given in (12). 


\section{APPENDIX B}

\section{DERIVATION OF THE UPDATE FORMULA (9)}

In order to derive the final update equation (9), we note that the above computations only depend on the variation with respect to the image argument, $I$. Thus including the kernel function $\omega(x, y)$ as in equation (6) would yield the exact same computation. So the first variation of (6) is simply

$$
\begin{gathered}
\delta E_{h, \omega, \tilde{I}_{l}}(I)=\frac{2}{|\Omega|} \int_{\Omega} \omega(x, y) \operatorname{sign}\left(I(x)-\tilde{I}_{l}(y)\right) d y \\
-\frac{2}{|\Omega|} \int_{\Omega} \omega(x, y) \operatorname{sign}(I(x)-I(y)) d y
\end{gathered}
$$

Coupling this with the first variation of (7) which is $\delta E_{g, I_{s}}(I)=\kappa_{I_{s}}(x)-\kappa_{I}(x)$, where $\kappa_{I}(x)$ is the Euclidean curvature of the level line of $I$ at location $x$, the update equation (9) is obtained via gradient descent on the total energy (8). $\frac{d E(I(x, t))}{d t} \leq 0$ for $I(x, t)$ satisfying (9), and the solution of (9) approaches the minimizer of (8).

\section{REFERENCES}

[1] A. Buades, B. Coll, and J. M. Morel, "A review of image denoising algorithms, with a new one," Multiscale Model. Simul., vol. 4, no. 2, pp. 490-530, 2005.

[2] R. Fattal, M. Agrawala, and S. Rusinkiewicz, "Multiscale shape and detail enhancement from multi-light image collections," ACM Transactions on Graphics, vol. 26, no. 3, p. 51, 2007.

[3] L. Yuan, J. Sun, L. Quan, and H.-Y. Shum, "Image deblurring with blurred/noisy image pairs," IACM Transactions on Graphics, vol. 26, no. 3, pp. 1-10, 2007.

[4] — - "Progressive inter-scale and intra-scale non-blind image deconvolution," in SIGGRAPH 'O8: ACM SIGGRAPH 2008 papers. New York, NY, USA: ACM, 2008, pp. 1-10.

[5] F. Sroubek and J. Flusser, "Multichannel Blind Iterative Image Restoration,” IEEE Transactions on Image Processing, vol. 12, no. 9, pp. 1094-1106, 2003.

[6] _ - "Multichannel Blind Deconvolution of Spatially Misaligned Images," IEEE Transactions on Image Processing, vol. 14, no. 7, p. 874883, 2005.

[7] J. Telleen, A. Sullivan, J. Yee, O. Wang, P. Gunawardane, I. Collins, and J. Davis, "Synthetic shutter speed imaging," in EUROGRAPHICS, 2007, pp. 522 - 529.

[8] A. Buades, Y. Lou, J. M. Morel, and Z. Tang, “A note on multi-image denoising," Proceedings of the International Workshop on Local and Non-Local Approximation (LNLA) in Image Processing, August 2009.

[9] L. Zhang, A. Deshpande, and X. Chen, “Denoising vs. deblurring: Hdr imaging techniques using moving cameras," in IEEE Conference on Computer Vision and Pattern Recognition, 2010, pp. 522 - 529.

[10] G. Petschnigg, M. Agrawala, H. H., R. Szeliski, M. Cohen, and K. Toyama, "Digital photography with flash and no-flash image pairs," ACM Transactions on Graphics, vol. 23, no. 3, pp. 664-672, 2004.

[11] E. Eisemann and F. Durand, "Flash photography enhancement via intrinsic relighting," ACM Transactions on Graphics (TOG), vol. 23, no. 3, pp. 673-678, 2004.

[12] J. Kopf, M. F. Cohen, D. Lischinski, and M. Uyttendaele, “Joint bilateral upsampling," ACM Transactions on Graphics (Proceedings of SIGGRAPH 2007), vol. 26, no. 3, p. to appear, 2007. 
[13] S. Zhuo, D. Guo, and T. Sim, "Robust flash deblurring," in CVPR, 2010, pp. 2440-2447.

[14] D. Krishnan and R. Fergus, "Dark flash photography," ACM Trans. Graph., vol. 28, no. 3, 2009.

[15] H. J. Seo and P. Milanfar, "Robust flash denoising/deblurring by iterative guided filtering," EURASIP J. Adv. Sig. Proc., vol. 2012, p. 3, 2012 .

[16] J. Rabin, J. Delon, and Y. Gousseau, "Regularization of transportation maps for color and contrast transfer," in Image Processing (ICIP), 2010 17th IEEE International Conference on. IEEE, 2010, pp. 1933-1936.

[17] N. Papadakis, E. Provenzi, and V. Caselles, "A variational model for histogram transfer of color images," Image Processing, IEEE Transactions on, no. 99, pp. 1-1, 2011.

[18] T. Huang and H. Chen, "Landmark-based sparse color representations for color transfer," in Computer Vision, 2009 IEEE 12th International Conference on. IEEE, 2009, pp. 199-204.

[19] J. Jia, J. Sun, C.-K. Tang, and H.-Y. Shum, "Bayesian correction of image intensity with spatial consideration," in Computer Vision ECCV 2004, ser. Lecture Notes in Computer Science, T. Pajdla and J. Matas, Eds. Springer Berlin / Heidelberg, 2004, vol. 3023, pp. $342-354$.

[20] M. Bertalmío and S. Levine, "Fusion of Bracketing Pictures," in Visual Media Production, 2009. CVMP'09. Conference for. IEEE, 2009, pp. 25-34.

[21] I. Cox, S. Hingorani, S. Rao, and B. Maggs, "A maximum likelihood stereo algorithm," Computer vision and image understanding, vol. 63 , no. 3, pp. 542-567, 1996.

[22] T. Mertens, J. Kautz, and F. V. Reeth, "Exposure fusion,” in Pacific Conference on Computer Graphics and Applications, 2007, pp. 382-390.

[23] E. A. Khan, A. O. Akyüz, and E. Reinhard, "Ghost removal in high dynamic range images," in ICIP, 2006, pp. $2005-2008$.

[24] P.-Y. Lu, T.-H. Huang, M.-S. Wu, Y.-T. Cheng, and Y.-Y. Chuang, "High dynamic range image reconstruction from hand-held cameras," in $C V P R, 2009$, pp. 509-516.

[25] M. Tico, N. Gelfand, and K. Pulli, "Motion-blur-free exposure fusion," in Image Processing (ICIP), 2010 17th IEEE International Conference on. IEEE, 2010, pp. 3321-3324.

[26] G. Sapiro and V. Caselles, "Histogram modification via differential equations," J. Differential Equations, vol. 135, no. 2, pp. 238-268, 1997.

[27] M. Bertalmío, V. Caselles, E. Provenzi, and A. Rizzi, "Perceptual color correction through variational techniques," IEEE Trans. Image Process., vol. 16, no. 4, pp. 1058-1072, 2007.

[28] "http://www.revisionfx.com/products/twixtor/."

[29] Y. Shinagawa and T. Kunii, "Unconstrained automatic image matching using multiresolutional critical-point filters," IEEE Transactions on Pattern Analysis and Machine Intelligence, pp. 994-1010, 1998.

[30] N. Papenberg, A. Bruhn, T. Brox, S. Didas, and J. Weickert, "Highly accurate optic flow computation with theoretically justified warping," International Journal of Computer Vision, vol. 67, no. 2, pp. 141-158, 2006.

[31] J. Delon and A. Desolneux, "Stabilization of flicker-like effects in image sequences through local contrast correction," SIAM Journal on Imaging Sciences, vol. 3, p. 703, 2010.

[32] C. Ballester, M. Bertalmío, V. Caselles, G. Sapiro, and J. Verdera, "Filling-in by joint interpolation of vector fields and gray levels," IEEE Trans. Image Process., vol. 10, no. 8, pp. 1200-1211, 2001.

[33] M. Bertalmío and S. Levine, "Denoising an image by denoising its curvature image,” in In preparation., 2013.

[34] E. Reinhard, M. Adhikhmin, B. Gooch, and P. Shirley, “Color transfer between images," Computer Graphics and Applications, IEEE, vol. 21 , no. 5 , pp. $34-41,2001$

[35] E. Reinhard and T. Pouli, "Colour spaces for colour transfer," Computational Color Imaging, pp. 1-15, 2011. 
[36] L. Rudin, S. Osher, and E. Fatemi, "Nonlinear total variation based noise removal algorithms," Physica D: Nonlinear Phenomena, vol. 60, no. 1-4, pp. 259-268, 1992.

[37] J.-M. M. Antoni Buades, Bartomeu Coll, “Non-local Means Denoising," Image Processing On Line, 2011. 